\title{
ChemComm
}

\section{Probing morphological changes in polymersomes with magnetic birefringence $\dagger$}

Cite this: Chem. Commun., 2014, 50,5394

Received 30th September 2013, Accepted 24th October 2013

DOI: $10.1039 / c 3 c c 47483 f$

www.rsc.org/chemcomm

\author{
Roger S. M. Rikken, ${ }^{a b}$ Harmen H. M. Kerkenaar, ${ }^{a b}$ Roeland J. M. Nolte, ${ }^{b}$ \\ Jan C. Maan, ${ }^{a}$ Jan C. M. van Hest, ${ }^{b}$ Peter C. M. Christianen ${ }^{a}$ and \\ Daniela A. Wilson ${ }^{\mathrm{b}}$
}

\begin{abstract}
Magnetic birefringence was used for in situ monitoring of the morphological changes in diamagnetic polymersomes during shapetransformation by dialysis. The birefringence was found to be very sensitive to the polymersome morphology, as determined by electron microscopy. The deflation of polymersomes into disks was observed, followed by a bending and partial inflation into stomatocytes.
\end{abstract}

Amphiphilic block-copolymers can self-assemble in water into bilayer vesicles named polymersomes. ${ }^{1}$ Many properties of these polymersomes, such as flexibility, permeability and functionality, can be tuned by varying the type or length of either the hydrophobic or the hydrophilic part of the block-copolymer ${ }^{1}$ or by adding functional groups to make them stimuli responsive. ${ }^{2}$ Flexibility and permeability can also be affected by the addition of an organic solvent, such as tetrahydrofuran (THF), which acts as a plasticizer for the hydrophobic part of the polymersome membrane. ${ }^{3,4}$ It has been previously demonstrated that polymersomes, self-assembled from poly(ethylene glycol)-polystyrene (PEG-PS) in a mixture of THF, 1,4-dioxane and water, undergo shape transformations into bowl-shaped structures called stomatocytes by dialysis against pure water. $^{5 a}$ Also the conformation of these stomatocytes could be further manipulated by reverse dialysis against a mixture of water, THF and dioxane. ${ }^{5 b}$ This control over morphology has led to nanoparticle encapsulation by the stomatocytes ${ }^{6}$ and their supramolecular assembly to give stomatocyte nanorockets. ${ }^{7}$ These properties make polymersomes and stomatocytes very promising candidates as nanocontainers in drug delivery or nanochemistry.

Until now, the effect of dialysis on the morphology of the polymersomes or stomatocytes has been investigated by taking

\footnotetext{
${ }^{a}$ High Field Magnet Laboratory (HFML), Radboud University Nijmegen, Toernooiveld 7, 6525 ED Nijmegen, The Netherlands. E-mail: p.christianen@science.ru.nl

${ }^{b}$ Institute of Molecules and Materials, Radboud University Nijmegen,

Heyendaalseweg 135, 6525 AJ, Nijmegen, The Netherlands.

E-mail:d.wilson@science.ru.nl

$\dagger$ Electronic supplementary information (ESI) available: Materials and instrumentation, polymersome sample preparation, statistics on points c, e and h, DLS and TEM/SEM-images at points a-h on the birefringence curve. See DOI: 10.1039/ c3cc $47483 f$
}

samples at regular intervals followed by the ex situ imaging of their conformations using electron microscopy. Here, we demonstrate how the morphology of polymersomes during dialysis can be probed in a continuous and non-invasive way using in situ magnetic birefringence. These measurements clearly reveal the exact times at which the morphology of the polymersomes change, making it possible to take samples for electron microscopy at the crucial points of dialysis.

Magnetic birefringence can be observed upon the alignment of molecules or aggregates. Even seemingly non-magnetic matter (like the vast majority of polymers and biomolecules) is in fact weakly magnetic (diamagnetic). Molecules used for self-assembly are usually anisotropic in shape, leading to a magnetic response that is also anisotropic. These molecules therefore have a preferential orientation in a magnetic field. The difference in energy between two orthogonal orientations of a molecule is normally quite small and hence the alignment is largely randomized by thermal motion. However, when these molecules form aggregates or self-assemble into supramolecular structures, the total diamagnetic anisotropy can be enhanced significantly. ${ }^{8}$ This principle has been used to orient organic nanostructures composed of a wide variety of molecules, including polymers. ${ }^{9-12}$ The diamagnetic anisotropy, and hence the magnetic alignment, of these self-assembled structures is also related to their overall shape. For instance, a sphere with an isotropic orientational distribution of molecules has no preferential axis of alignment. However, when the distribution of molecular orientations is anisotropic magnetic alignment can occur. For example, a disk constructed from diamagnetic anisotropic molecules aligns with its surface either parallel or perpendicular to the magnetic field, depending on the sign of the diamagnetic anisotropy of the molecular building blocks.

Magnetic orientation of supramolecular aggregates in solution results in a difference in the refractive index for light polarized parallel and perpendicular with respect to the magnetic field. This magnetic birefringence has been frequently measured to determine the degree of alignment of various aggregates in magnetic fields. ${ }^{9-12}$ In this communication, we use magnetic birefringence to probe morphological changes in polymersomes during dialysis, while 
TOP VIEW

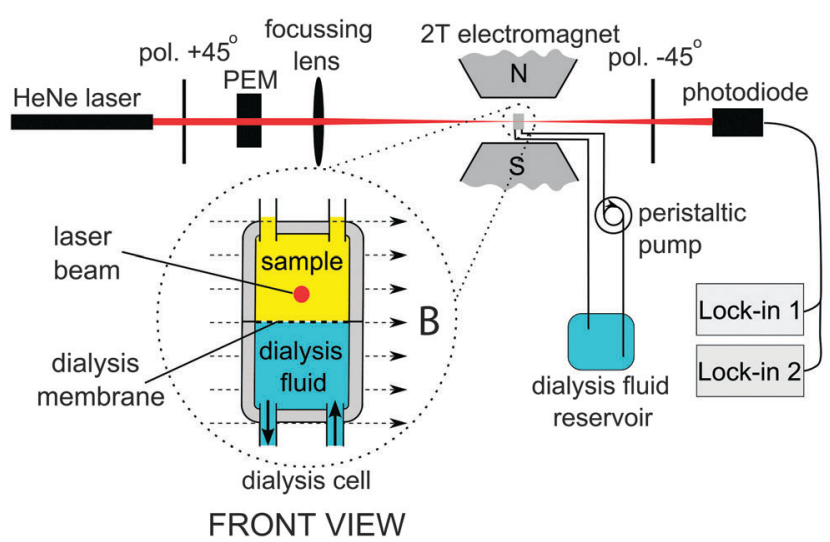

Fig. 1 Experimental setup. The dialysis cell is placed between the poles of a $2 \mathrm{~T}$ electromagnet. The close-up of the dialysis flow cell shows how the two chambers are separated by a $12-14 \mathrm{kDa}$ cut-off membrane. The laser beam is directed through the upper chamber containing the polymersome sample. The birefringence is detected using a standard polarization modulation technique, using a photo-elastic modulator (PEM) and crossed polarizers (pol.). The magnetic field $(B)$ lines are indicated by the dashed arrows pointing right. The dialysis fluid is refreshed continuously as indicated by the two solid arrows.

further insight into the mechanism of osmotically induced shape change is presented. To explore the morphological changes we implemented a dialysis cell with a magnetic birefringence setup in a 2 T magnet (Fig. 1). Light of a HeNe laser (1.5 mW, $632.8 \mathrm{~nm}$ ) was focussed on the upper chamber of a flow cell, which contained a $\mathrm{PEG}_{44}-\mathrm{PS}_{133}$ polymersome sample. Initially the sample consisted of spherical polymersomes in pure water, having a radius of $252 \mathrm{~nm}$ and a PDI of 0.134 , as determined by dynamic light scattering (DLS). A dialysis fluid consisting of 50\% water, $40 \%$ THF and $10 \%$ 1,4-dioxane was pumped through the bottom chamber at a rate of $100 \mathrm{~mL} \mathrm{~h}^{-1}$. The chambers were separated by a $12-14 \mathrm{kDa}$ cut-off membrane. The magnetic birefringence was detected using a standard polarization modulation technique. ${ }^{13,14}$

The birefringence was measured over a time interval of 360 minutes (blue curve in Fig. 2I). At the beginning of the experiment, the birefringence remained zero up to 170 minutes (point c), after which a rapid increase in the signal was observed. A maximum was reached at 230 minutes (point e), after which the birefringence decreased. From point $\mathrm{g}$ onwards, the sample showed a small birefringence, which remained constant until the end of the measurement (point $h$ ). At certain time points, magnetic field sweeps were performed where the magnetic field was reduced to zero and subsequently brought back to $2 \mathrm{~T}$ (indicated in Fig. 2I by the coloured lines 1-5). The field sweeps were sufficiently rapid (about one minute) to allow the subsequent birefringence signal to remain unchanged by the continuous dialysis. The birefringence always decreased to zero when the magnetic field was brought to zero. Restoring the field to $2 \mathrm{~T}$ also resulted in recovery of the birefringence to the same value before the field sweep, showing that the measured signal is not caused by drift. The inset of Fig. 2I shows the birefringence as a function of $B^{2}$. All curves scale quadratically with the applied magnetic field, implying a non-saturated magnetic alignment. This is usually observed in the low magnetic field regime where the competition with thermal motion tends to randomize the orientation of the molecules resulting in partial alignment with the field.
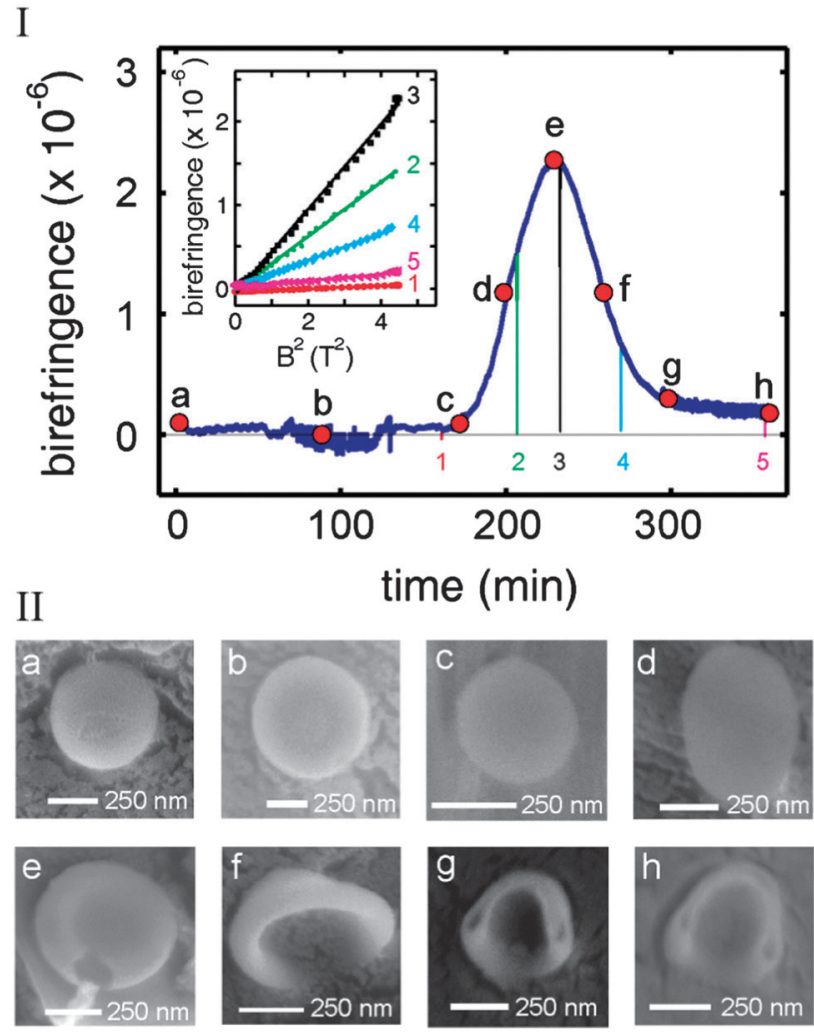

Fig. 2 (I) Magnetic birefringence during dialysis of a polymersome sample at $2 \mathrm{~T}$. Several magnetic field sweeps (1-5) were performed, which are plotted against $B^{2}$ in the inset. Samples taken at points a- $\mathrm{h}$ were investigated by cryoSEM, images of which are shown in (II) $(a-h)$. Note that the birefringence increases from the moment the polymersomes start to deflate and become anisotropically shaped (c-e). The decrease in birefringence occurs when the disks at point e fold and partly inflate to form stomatocytes (f-h).

Measuring magnetic birefringence during dialysis offers the opportunity to stop dialysis at well-defined points to take samples for further investigation by electron microcopy. In this manner, the morphology can be related to the amplitude of the birefringence signal. Samples were taken at points $a-h$ as indicated by the red dots in Fig. 2I (ESI $\dagger$ ).

Cryogenic-Scanning Electron Microscopy (cryo-SEM) images of samples (a-h) are shown in Fig. 2II. At the beginning of dialysis, polymersomes were present with the expected spherical morphology (point a) corresponding to zero birefringence. At points b and c, no changes in conformation were observed, in agreement with the measured constant magnetic birefringence. At point $d$ the birefringence increased to half its maximal value. The cryo-SEM images show ellipsoidal polymersomes, which can only be explained by a partial deflation of the spherical polymersomes. When the birefringence reached a maximum (point e), flat disks were observed under a cryo-SEM. Continuation of the dialysis led to the bending of the flat disks (point $f$ ) and the formation of stomatocytes (points $g$ and $\mathrm{h}$ ), where the structures partly inflated again. At this point, the hydrodynamic radius of the polymersomes was decreased to $218 \mathrm{~nm}$ with a PDI of 0.12, as determined by DLS (see ESI + ).

At all points, the magnitude of the birefringence reflects the shape of the structures. All spherical polymersomes show zero 


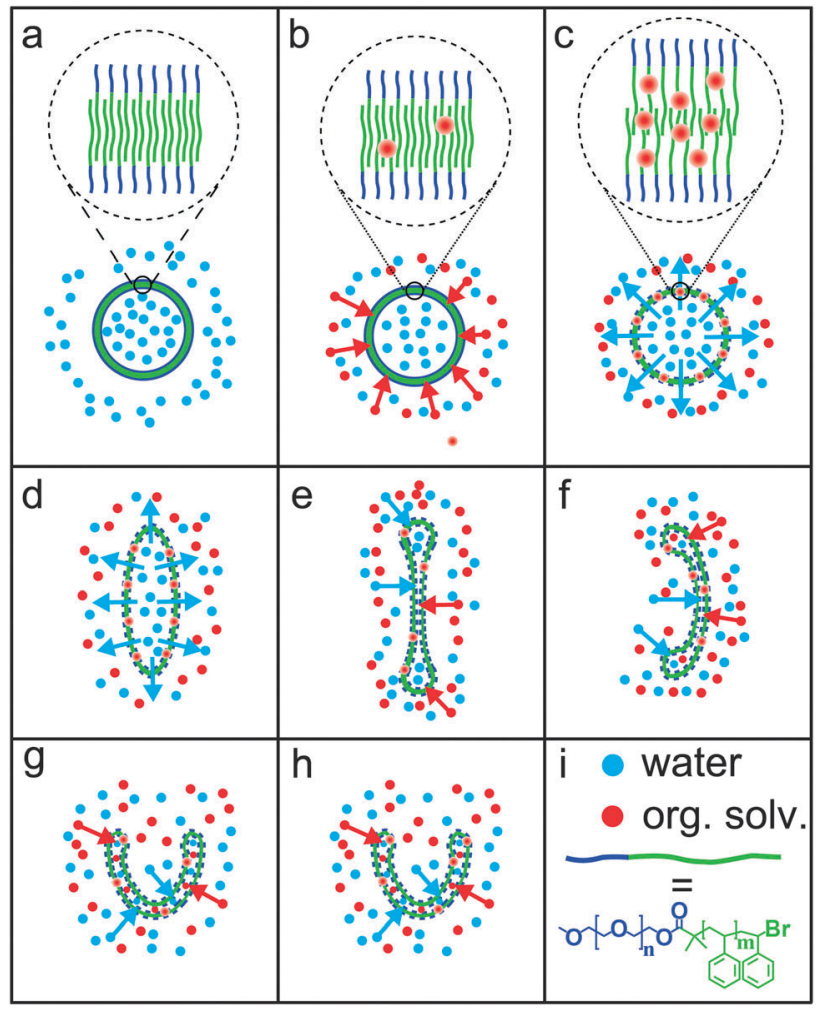

Scheme $1(a-h)$ Proposed mechanism of the dialysis of polymersomes against 50\% water, 40\% THF and 10\% 1,4-dioxane. The morphologies correspond to those shown in Fig. $211 . \ln (a-c)$, the polymersome membrane is shown enlarged. The organic solvent acts as a plasticiser, which swells the membrane. Swollen membranes are indicated by dashed lines in figures $(c-h)$ compared to the solid lines in figures (a) and (b). (i) Red and blue dots represent organic solvent and water, respectively. The block co-polymer is drawn schematically as a short blue line (PEG) connected to a longer green line (PS).

birefringence since they cannot be aligned. ${ }^{15}$ With increasing deflation of the polymersomes the birefringence amplitude increases. The largest birefringence is observed for disks, in which most of the polymers have identical orientation, perpendicular to the flat surface. The transition of disks to stomatocytes leads to a more curved conformation, and hence a smaller diamagnetic anisotropy.

To explain the effects of dialysis on the morphology of the polymersomes, we propose the mechanism depicted in Scheme 1. At the starting point of the dialysis, the polymersome membrane is very rigid, due to lack of a plasticising organic solvent (a). During dialysis, the organic solvent (red dots) enters the polymersome membrane (b). At point c, enough organic solvent has entered the hydrophobic part of the polymersome membrane to make it permeable to water (blue dots). From this point on, water diffuses out of the polymersome, as a result of the concentration gradient over the membrane. The outflow of water leads to a reduced inner volume, giving rise to an elongated shape (d). Further outflow of water eventually leads to a disk, which shows the highest birefringence (e). At this point the disk begins to bend in one direction, most probably due to fluctuations in the membrane that give rise to a spontaneous curvature. The membrane starts to fill slowly with the mixture of water and organic solvent again (f). This process continues and stomatocytes are formed $(\mathrm{g}, \mathrm{h})$.
In conclusion, we have demonstrated that magnetic birefringence can be used as a useful tool to monitor morphological changes in polymersomes resulting from dialysis in a flow cell. This method has the advantage of being non-invasive; while the morphology can be determined without disrupting the dialysis setup for sample investigation by electron microscopy. Also, because the dialysis in the flow cell is rather slow (in the order of several hours), the shape transformations can be determined at very precise points on the birefringence curve, providing samples with very specific and predictable conformations. In principle, this method could be extended to obtain more quantitative information about the rigidity of the structures in all stages of the process. However this would require a more extended theoretical analysis.

We acknowledge the support of the HFML-RU/FOM, member of the European Magnetic Field Laboratory (EMFL). R. S. M. Rikken acknowledges the Graduate School for Molecules and Materials for a PhD position and NWO for its corresponding grant. D. A. Wilson acknowledges funding from the European Research Council under the European Union's Seventh Framework Programme (FP7/20072012)/ERC-StG 307679 "StomaMotors". R. J. M. Nolte acknowledges support from the European Research Council (ERC Advanced grant 290886-ALPROS) and R. J. M. Nolte and J. C. M. van Hest acknowledge support from the Ministry of Education, Culture and Science (Gravity program 024.001.035). Finally, we would like to thank G.-J. Janssen for his assistance with the electron microscopes and P. Walraven for the fabrication of the flow cell.

\section{Notes and references}

1 D. E. Discher and F. Ahmed, Annu. Rev. Biomed. Eng., 2006, 8, 323.

2 F. Meng, Z. Zhong and J. Feijen, Biomacromolecules, 2009, 10, 197.

3 S. J. Holder and N. A. J. M. Sommerdijk, Polym. Chem., 2011, 2, 1018.

4 Y. Yu and A. Eisenberg, J. Am. Chem. Soc., 1997, 119, 8383-8384.

5 (a) K.-T. Kim, J. Zhu, S. A. Meeuwissen, J. J. L. M. Cornelissen, D. J. Pochan, R. J. M. Nolte and J. C. M. van Hest, J. Am. Chem. Soc., 2010, 132, 12522; (b) S. A. Meeuwissen, K. Kim, Y. Chen, D. J. Pochan and J. C. M. van Hest, Angew. Chem., Int. Ed., 2011, 50, 7070.

6 D. A. Wilson, R. J. M. Nolte and J. C. M. van Hest, J. Am. Chem. Soc., 2012, 134, 9894.

7 (a) D. A. Wilson, R. J. M. Nolte and J. C. M. van Hest, Nat. Chem., 2012, 4, 268; (b) D. A. Wilson, B. de Nijs, A. van Blaaderen, R. J. M. Nolte and J. C. M. van Hest, Nanoscale, 2013, 5, 1315.

8 R. Rikken, R. J. M. Nolte, J. C. Maan, J. C. M. van Hest, D. A. Wilson and P. C. M. Christianen, Soft Matter, 2013, DOI: 10.1039/C3SM52294F.

9 I. O. Shklyarevskiy, M. I. Boamfa, P. C. M. Christianen, F. Touhari, H. van Kempen, G. Deroover, P. Callant and J. C. Maan, J. Chem. Phys., 2002, 116, 8407.

10 P. C. M. Christianen, I. O. Shklyarevskiy, M. I. Boamfa and J. C. Maan, Phys. Biol., 2004, 346-347, 255.

11 J. C. Gielen, M. Wolffs, G. Portale, W. Bras, O. Henze, A. F. M. Kilbinger, W. J. Feast, J. C. Maan, A. P. H. J. Schenning and P. C. M. Christianen, Langmuir, 2009, 25, 1272.

12 J. C. Gielen, I. O. Shklyarevskiy, A. P. H. J. Schenning, P. C. M. Christianen and J. C. Maan, Sci. Technol. Adv. Mater., 2009, 10, 014601.

13 G. Maret and K. Dransfeld, Strong and Ultrastrong Magnetic Fields and Their Applications, Springer-Verlag, Berlin, 1985, ch. 4.

14 J. C. Kemp, Polarized Light and its Interaction with Modulated Devices A Methodology Review, HINDS International Inc., Hillsboro, 1987.

15 This indicates that the PEG-PS polymersomes are not yet deformed at $2 \mathrm{~T}$. Magnetic deformation of other nanocapsules has been observed at higher magnetic fields up to 20 T: I. O. Shklyarevskiy, P. Jonkheijm, P. C. M. Christianen, A. P. H. J. Schenning, E. W. Meijer, O. Henze, F. M. Kibinger, W. J. Feast, A. Del Guerzo, J. P. Desvergne and J. C. Maan, J. Am. Chem. Soc., 2005, 127, 1112. 